\title{
Chatter Identification of Three-Dimensional Elliptical vibration Cutting Process Based on Empirical Mode Decomposition and Feature Extraction
}

\author{
Mingming Lu ${ }^{1} \oplus$, Bin Chen ${ }^{1}$, Dongpo Zhao ${ }^{1}$, Jiakang Zhou ${ }^{1}$, Jieqiong Lin ${ }^{1, *}$, Allen Yi ${ }^{2}$ \\ and Hao Wang ${ }^{1}$ \\ 1 Key Laboratory of Micro-Nano and Ultra-precision Manufacturing of Jilin Province, \\ School of Mechatronic Engineering, Changchun University of Technology, Changchun 130012, China; \\ lumm@ccut.edu.cn (M.L.); chen0202130612@163.com (B.C.); dpzhao1@163.com (D.Z.); \\ zhoujiakang07@163.com (J.Z.); wanghao9439@163.com (H.W.) \\ 2 Department of Industrial, Welding and Systems Engineering, Ohio State University, \\ 210 Baker Systems Building, 1971 Neil Avenue, Columbus, OH 43210, USA; yi.71@osu.edu \\ * Correspondence: linjieqiong@ccut.edu.cn; Tel.: +86-13756067918
}

Received: 13 November 2018; Accepted: 14 December 2018; Published: 21 December 2018

\begin{abstract}
Three-dimensional elliptical vibration cutting (3D-EVC) is one of the machining methods with the most potential in ultra-precision machining; its unique characteristics of intermittent cutting, friction reversal, and ease of chip removal can improve the machinability of materials in the cutting processes. However, there is still not much research about the chattering phenomenon in the 3D-EVC process. Therefore, based on the empirical mode decomposition (EMD) technique and feature extraction, a chatter identification method for 3D-EVC is proposed. In 3D-EVC operations, the vibration signal is collected by the displacement sensors and converted to frequency domain signal by fast Fourier transform (FFT). To identify tool cutting state using the vibration frequency signal, the vibration signals are decomposed using empirical mode decomposition (EMD), a series of intrinsic mode functions (IMFs), so the instantaneous frequency can be reflected by the vibration signals at any point. Then, selecting the primary IMFs which contain rich chatter information as the object in feature extraction identification, and two identification indexes, that is, the mean square frequency and self-correlation coefficient, are calculated for the primary IMFs by MATLAB software, to judge the chatter phenomenon. The experimental results showed that the mean square frequency and self-correlation coefficient of the three cutting states increase with the increase in the instability of the cutting state. The effectiveness of the improved chatter recognition method in 3D-EVC machining is verified.
\end{abstract}

Keywords: chatter identification; three-dimensional elliptical vibration cutting; empirical mode decomposition; intrinsic mode function; feature extraction

\section{Introduction}

Three-dimensional elliptical vibration cutting (3D-EVC) is an ultra-precision cutting technology with significant development potential. Its unique characteristics of intermittent cutting, friction reversal, and ease of chip removal can improve the machinability of difficult-to-cut materials in the cutting process [1-3]. Since 3D-EVC technology was first introduced in 2005, the research field has mainly focused on the mechanism of cutting, the design of 3D-EVC apparatus, path planning of the tool, specific applications, and so on. [4,5]. At present, how to study the chatter phenomenon in the process of non-resonance 3D-EVC machining is an important problem. In the ultra-precision cutting technology processes, the main reasons that the effect of unstable vibration could be reduced is by the 
increase of the spindle speed, small cutting depth, and great performance of the diamond tool. But the damage accompanying chatter is mainly reflected in reducing the accuracy of the work-piece of the optical parts, increasing the surface roughness of the work-piece, speeding up the wear on the cutting tools, and causing damage to the spindle of the machine tool [6,7].

EVC is an intermittent cutting technology, which has the characteristics of friction reversal, tool wear suppression, and easy chip removal. However, there is no specific literature on the identification of chatter in the 3D-EVC cutting processes [8]. In 2011, Ma et al. proposed that the two-dimensional EVC technology had a certain inhibitory effect in chatter suppression compared to the traditional cutting method, and the effectiveness of the effect of chatter suppression was verified by experiment, but this was limited to two-dimensional elliptical vibration cutting [9]. In 2016, Jung et al. collected the real-time signal from the 2D-EVC process and analyzed the chatter phenomenon according to the frequency domain analysis and surface of the work-piece [10]. They obtained the friction chatter mechanism of the plough force during the initial period of each ellipse movement cycle and suppressed the chatter vibration by changing the guiding angle in 2D-EVC process. It was also the earliest research on identification and suppression of the chatter phenomenon in the 2D-EVC cutting processes. Therefore, exploring a new method to identify the chattering phenomenon applied to the 3D-EVC processes is critical to improving the machining accuracy of the 3D-EVC technology.

In the field of ultra-precision cutting, research on chatter is focused on the influence of undesired vibration, the chatter identification and the modeling method of chatter mechanism, and so on. Chatter is a poor vibration phenomenon in metal processing, which has effects on the machined work-piece surface quality, reduced cutting efficiency and productivity, as well as producing a lot of noise, and so on. The mechanical vibration has three forms: free vibration, forced vibration, and self-excited vibration. The free vibration is caused by impact during the machining process. The forced vibration is mainly caused by the unbalance effect of the machine tool components. The free vibration and forced vibration are relatively easy to identify and eliminate. However, the self-excited vibration is a significant factor affecting cutting stability. Zhang et al. proposed the physical modes of vibration, in ultra-precision machining, and the effect on the generated surface is related to the many factors, including the properties of materials, cutting conditions and the relative vibration between tool and work-piece [11]. Chen et al. presented the mathematical models for modeling and analyzing the vibration and surface roughness in precision turning, and also established a relationship model between tool vibration and surface profile and roughness in the precision end-milling process [11,12]. A simple method for the detection of milling chatter was presented by Zhang. The assessment of milling process stability by recursive drawing method combined with Hilbert Huang transform was proposed by Rafal [13]. In addition, with the development of the sensor and the modern signal processing technology, chatter identification technology has been greatly improved. Various signals have been applied in chatter identification, such as cutting force [14-16], sound [17] and the acceleration [18,19]. Cao et al. presented an effective chatter identification method based on the two advanced signals processing techniques, wavelet package transform (WT) and Hilbert-Huang transform (HHT), and the experimental results proved that the method can identify the chatter effectively [20]. In 2015, Cao et al. adopted a self-adaptive analysis method named ensemble empirical mode decomposition (EEMD), extracted two nonlinear indices as the indices of chatter symptoms, and presented a method for identifying the chattering phenomenon based on the two points in the end milling process. The results have shown that the mean value and standard deviation increase with the cutting state's instability [21,22].

The vibration signals belong to unsteady and nonlinear signals in the 3D-EVC process [23], traditional time-frequency analysis methods can analyze the global mean of the signal in the time-frequency domain, but it cannot analyze the feature information about the local area. In order to solve the lack of self-adaptability or poor adaptability in traditional time-frequency analysis methods, Huang et al. proposed a novel and adaptive decomposition method, which was named empirical mode decomposition (EMD), in 1998 [24]. Liu et al. decomposed the motor current signal into intrinsic mode functions (IMFs) and extracted the energy index and kurtosis index based on those IMFs for chatter 
detection [21]. Compared with the traditional time-frequency analysis, EMD is more appropriate for analyzing the non-stationary and nonlinear signals, as the EMD method can not only yield clear distribution for instantaneous frequency and amplitude, but also reflect the time-variation of the total energies, which is significant for the non-stationary and nonlinear signals. In recent years, the EMD method has been applied to signal processing, fault diagnosis [25] and pattern recognition [25,26].

Based on the above research, this paper presents chatter identification based on the empirical mode decomposition (EMD) technique and feature extraction. The method of chatter identification developed is divided into two parts: initial identification and feature extraction identification. In 3D-EVC operations, the vibration signals are collected by displacement sensors and converted to frequency domain signals by fast Fourier transform (FFT), and the cutting state of the whole machining system is determined according to the change of frequency. The vibration signals are decomposed by EMD, a series of intrinsic mode functions (IMFs), so the instantaneous frequency can be reflected by the vibration signals at any point. Selecting the primary IMFs which contain rich chatter information as the object in feature extraction identification, two identification indexes, that is, the mean square frequency (MSF) and self-correlation coefficient $\left(\rho_{1}\right)$, were calculated from the primary IMFs using MATLAB software to judge the chatter phenomenon. In addition, chatter identification is organized as follows. First step is the fast Fourier transform (FFT) analysis of the time domain signal to observe whether the frequency changes during the cutting process. If there is a frequency domain mutation, it indicated that chatter occurs during the cutting process. The second step is eigenvalue identification, which serves two purposes. One needs to test the chatter identification result of the initial step by using two distinct eigenvalue changes during the cutting process. More importantly, the eigenvalue can reflect the state change of the cutting process to a certain extent, which is used as a theoretical basis for the online identification of chatter.

This paper is structured as follows. Section 2 briefly introduces the signal processing method empirical mode decomposition (EMD), and describes the theory and strategy of the chatter identification. Then experimental setup and the cutting parameter setting are described in Section 3 . The results and discussions of the chatter identification method developed are given in Section 4. Finally, the conclusions are given in Section 5.

\section{Theory of Chatter Identification}

\subsection{Empirical Mode Decomposition}

With the rapid development of the time-frequency analysis, some signal processing technologies (such as short time Fourier transform, Wigner-Ville distribution and Wavelet transform) have the ability to analyze non-stationary and nonlinear signals. However, the methods of traditional time-frequency analysis have some limitations during analysis of non-stationary and nonlinear signals, such as a lack of adaptability. Compared with the methods of traditional time-frequency analysis, empirical mode decomposition (EMD) is more appropriate for analyzing the non-stationary and nonlinear signals. The EMD method can obtain clear instantaneous frequency and amplitude distribution and can reflect the time variation of total energy. This is very important for non-stationary and nonlinear signals. During the process of EMD, the vibration signal is processed smoothly, the fluctuations under different scales and the trend of the change are decomposed step by step, a series of data columns are generated with different characteristic scales, named the intrinsic mode functions (IMFs). The IMF obtained by the EMD is the approximate single-frequency signal which exists only at one frequency in every moment. It contains the real physical process in the vibration signals. In this method, the chatter phenomenon can be identified correctly by extracting the sensitive characteristic undesired vibration signal in each IMF.

In the process of EMD, if the local mean value is set to zero and has locally symmetric properties for a function or signal, then the essential condition of the mean instantaneous frequency is the same 
number of extreme points and zero-crossing points. Therefore, Huang et al. proposed the compact of the intrinsic mode function (IMF). These mode functions must satisfy the following two conditions:

(1) The number of extreme points and the number of zero-crossing points must be either equal or differ at most by one in the whole dataset.

(2) The local upper and lower envelope at any data point is symmetrical, which means the envelope of the local minimum value and the maximum value is zero.

The IMF reflects the inherent oscillatory of non-stationary and nonlinear signals, and there is only first -order oscillation mode in each of cycles. The phenomenon of mode mixing is nothingness. A typical of the IMF have the same extreme points and the number of zero-crossing points, at the same time, the upper and lower envelope is symmetrical about the time-axis, just exist a single frequency component at any time, as shown in Figure 1.

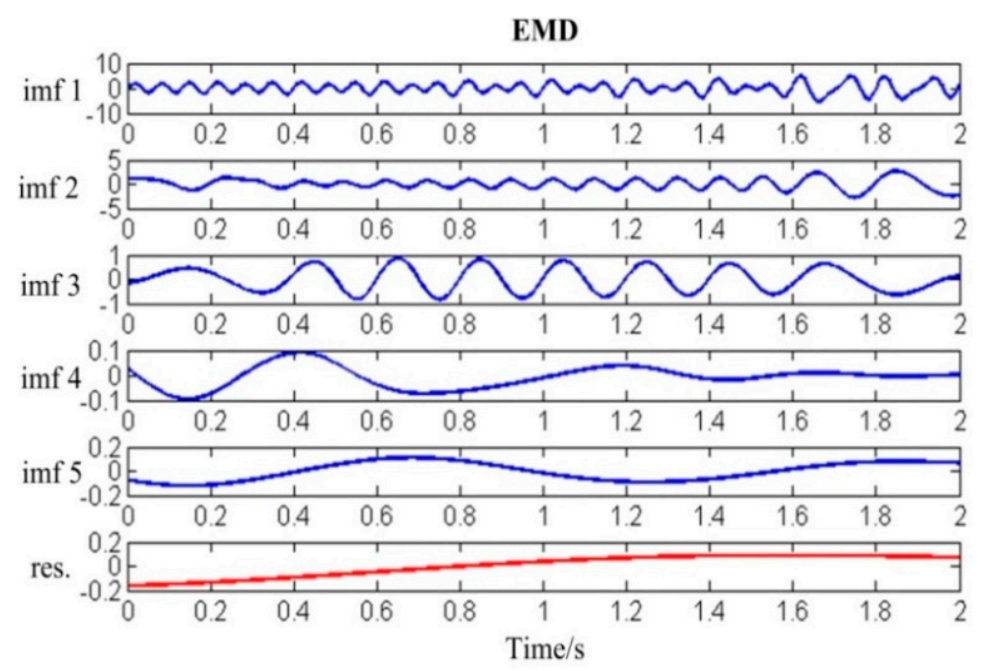

Figure 1. A series of intrinsic mode functions (IMF) obtained using empirical mode decomposition (EMD).

In addition, the EMD method which decomposes the signal is based on the following three assumptions:

(1) The signal has two extreme points at least-a maximum point and a minimum point.

(2) The characteristic time scale is defined as the time interval of the adjacent extreme points.

(3) If the signal does not have the extreme point but an inflection point exists, then derivation is performed one or more times to obtain the extreme points before decomposing the signal, and the results can be obtained by integrating the corresponding component.

The following is the brief description of the EMD algorithm:

First, calculating all local extreme point for a given signal $X(t)$, and forming the upper envelope by adopting the line cubic spline curve, and the lower envelope line also formed by the same way. The value of difference between $X(t)$ and the mean $m_{1}$ of upper (E1) and lower (E2) envelope is $h_{1}$. Then the new data $h_{1}$ are obtained as shown as follows:

$$
h_{1}=X(t)-m_{1}
$$

Regard $h_{1}$ as the new signal $X(t)$, and repeat the above steps until $h_{i}$ meets the two required conditions of IMFs, where $C_{1}$ represents the first order IMF sifting from the original signal. In general, the first order IMF contains the highest frequency component of the signal. 
Then obtain a difference signal $r_{1}$ which removes the high frequency component after separated $C_{1}$ from the original $X(t)$ :

$$
r_{1}=X(t)-C_{1}
$$

where $r_{1}$ is regarded as the new signal, and repeat the sifting process until the mean line between the upper and lower envelope line is close to zero at any point, denoted by $r_{n}$ :

$$
r_{n}=r_{n-1}-C_{n}
$$

Finally, $X(t)$ could be represented as the sum of IMFs and a residual function:

$$
X(t)=\sum_{j=1}^{n} C_{j}(t)+r_{n}(t)
$$

where $r_{n}(t)$ is a residual function which represents the average trend of the signal. The IMF component $C_{j}(t)$ represents the signal components from high to low in different frequency, respectively. The flowchart of the EMD algorithm as follows in Figure 2.

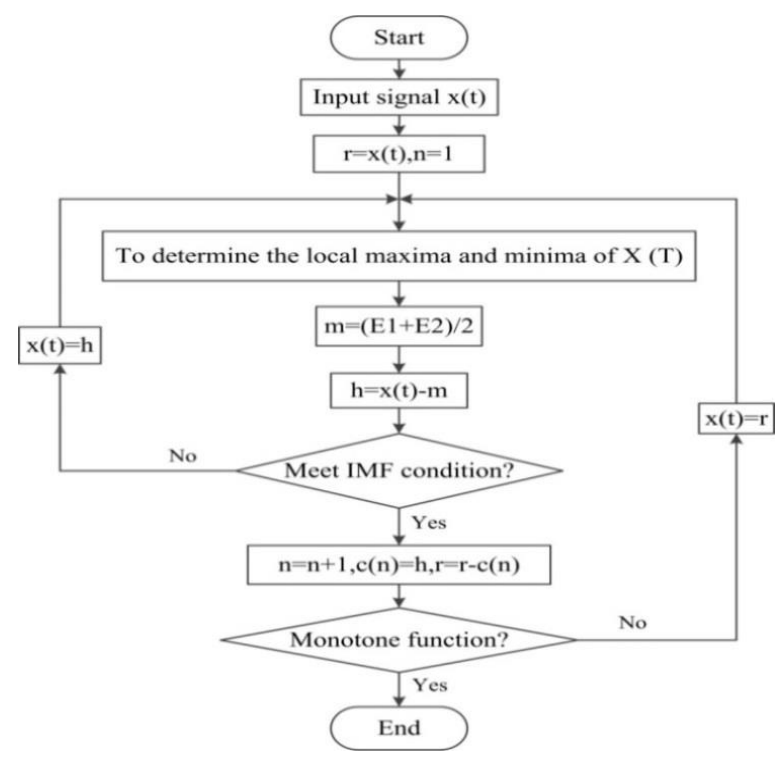

Figure 2. Flowchart of EMD algorithm.

\subsection{Principle of Chatter Identification}

Because of the inevitability of vibration in the cutting process, the displacement signals collected in the 3D-EVC processes are actually nonlinear and non-stationary signals. In the process of 3D-EVC, the selection of the chatter signal is significant for correctly identifying instability phenomena. In the process of the specific chatter identification experiment, the signal selected during the 3D-EVC process should follow the following two points: (1) the change of the state in the cutting process can be reflected from two aspects of the time domain and the frequency domain; and (2) the assembly of the sensor cannot affect the stability of the whole cutting system.

In the traditional machining process, the common signals applied in chatter identification are mainly the tool tip vibration displacement signal, the cutting force signal, the sound signal and the cutting acceleration signal. However, 3D-EVC technology is an ultra-precision manufacturing technology, and the cutting process has high precision as the small cutting rate, high cutting speed, and low cutting depth. In addition, the displacement sensor is easy to assemble with the tool without affecting the whole cutting system characteristics with high resolution. Therefore, the displacement 
sensor is used to measure the vibration and displacement signals of the tool tip as the main signal for chatter identification in the process of 3D-EVC.

In the process of 3D-EVC, the vibration frequency under a stable machining state is the frequency of the tool tip vibration set in the 3D-EVC apparatus. In order to avoid the phenomenon of over cutting or not cutting during the process, the vibration frequency of tool ellipse is relatively low. In this paper, the vibration frequency of the tool tip in the three directions is set as $40 \mathrm{~Hz}$, so the chatter frequency is higher than the original vibration frequency of the tool. When the cutting process becomes unstable during processing, the phenomenon of frequency shift will appear and the unstable frequency of chatter phenomenon during the cutting process will exceed the elliptical vibration frequency applied to the diamond tool in the non-resonant 3D-EVC apparatus. On the other hand, another important factor in the chatter identification strategy is the selection of chatter characteristics. There are many methods for extracting the features of the chatter state in the time domain and frequency domain. The mean square frequency (MSF) is an ideal characteristic value which could reflect the changing situation of the cutting signal in the frequency domain. MSF represents the weighted mean of the square of the vibration frequency, and the weight is the amplitude of the power spectrum. In the cutting process, the machining frequency is not a constant value due to the chatter appearance as a result of the change of cutting parameters, tool wear, the properties of the work-piece material and the processing mechanism under different processing methods, and so on. The chatter phenomenon will appear and the frequency of chatter gradually increase and exceed the elliptical vibration frequency applied to the diamond tool in the non-resonant 3D-EVC apparatus with the change of the cutting parameter. The formula of MSF is shown as the following:

$$
M S F=\sum_{i=2}^{N} x_{i}^{2} / 4 \pi^{2} \sum_{i=1}^{N} x_{i}^{2}
$$

In addition, the other parameter used for chatter identification is the one step self-correlation coefficient $\rho_{1}$. In general, $\rho_{1}$ has a consanguineous relationship with the variety of energy in vibration signals, the value of $\rho_{1}$ will reduce suddenly when the energy concentrates around the chatter frequencies when the amplitude of vibration signals becomes uneven with the chatter frequency. The value of $\rho_{1}$ can be expressed as:

$$
\rho_{1}=\cos 2 \pi f_{i} \Delta
$$

where $\Delta$ is the sampling interval, the value of $\rho_{1 i}$ decreased with the increase of $f_{i}$.

If the signal contains a variety of harmonic components, then the value of $\rho_{1}$ can be expressed as:

$$
\rho_{1}=\frac{\sum_{i=1}^{n} a_{i}{ }^{2} \cos 2 \pi f_{i} \Delta}{\sum_{i=1}^{n} a_{i}{ }^{2}}=\frac{\sum_{i=1}^{n} a_{i}{ }^{2} \rho_{1 i}}{\sum_{i=1}^{n} a_{i}{ }^{2}}
$$

In general, the conventional fast calculation method is shown as the following:

$$
\begin{gathered}
M S F=\frac{D}{4 \pi^{2} B} \\
\rho_{1}=\frac{C-A^{2}}{B-A^{2}}
\end{gathered}
$$

where, $A=\sum_{i=0}^{N} x_{i}, B=\sum_{i=0}^{N} x_{i}{ }^{2}, C=\sum_{i=0}^{N} x_{i} x_{i-1}, D=\sum_{i=0}^{N} x_{i}{ }^{2}$, respectively. $x_{i}(i=0,1,2, \ldots, N)$ represents the sample data, $\dot{x}_{i}=\frac{x_{i}-x_{i-1}}{\Delta}(i=1,2, \ldots, N)$ is one order difference, $N+1$ and $\Delta$ represents the sampling point and the sampling interval, respectively. 
Therefore, synthesizing two characteristic parameters of MSF and $\rho_{1}$, which represents the characteristics of vibration signal in the frequency domain, the changes of vibration states can be reflected accurately.

\subsection{The Flow Chart of Chatter Identification}

The flow chart of chatter identification in the 3D-EVC process is illustrated in Figure 3. Firstly, the driving signal is generated by Power PMAC controller (Delta Tau Data Systems, Inc., USA), and used for driving the piezoelectric stack in the cutting tool with the elliptical trajectory in three-dimensional space. The different vibration states can be obtained by changing the cutting parameters (the depth of cut, spindle speed or the feed rate). The vibration signal of the diamond tool in the processing of 3D-EVC was collected by the displacement sensors, and converted to frequency domain signal by fast Fourier transform (FFT), and the cutting state is determined (stable cutting, transient state and chatter state) according to the variation of frequency. On the other hand, the method of EMD is used for the vibration signals under various vibration states, selecting the sensitive IMFs which including the rich chatter information and calculate the value of MSF and $\rho_{1}$ corresponding to three cutting states. Finally, the chatter phenomenon in the 3D-EVC process can be identified correctly based on the two aspects of change characteristics.

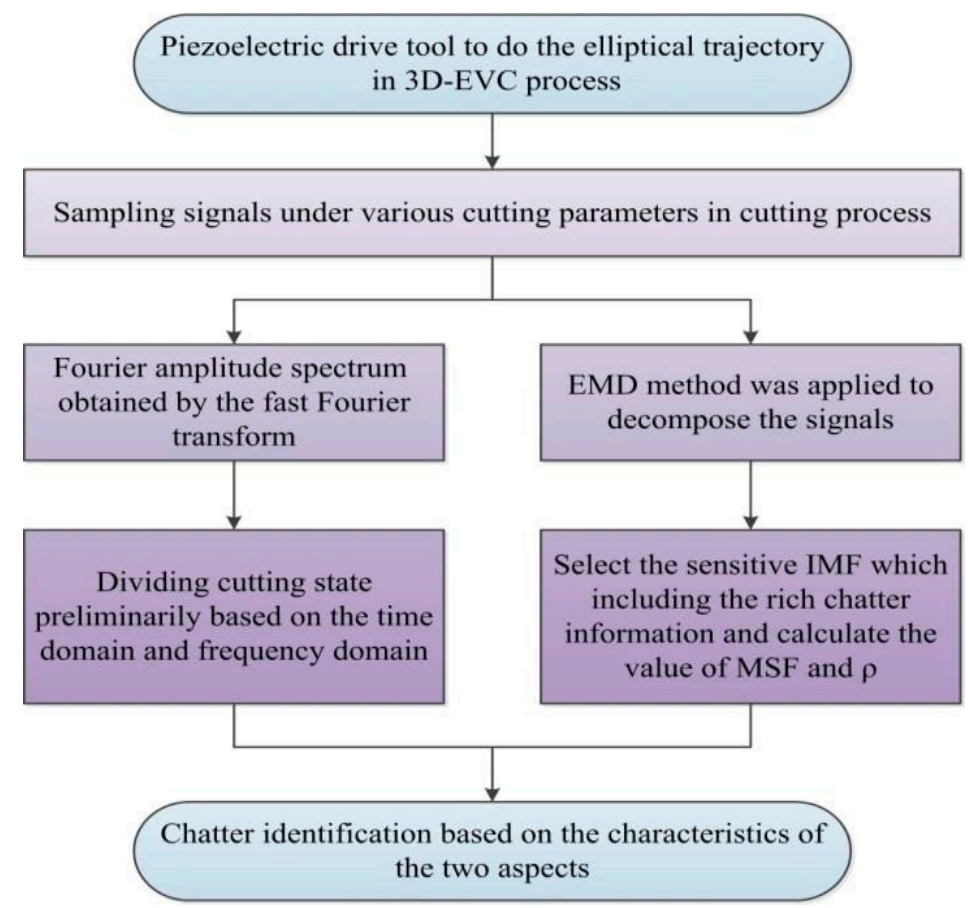

Figure 3. Flow chart of chatter identification in the three-dimensional elliptical vibration cutting (3D-EVC) process.

\section{Experimental Setup}

The proposed chatter identification method has been carried out in an ultra-precision machining machine, as shown in Figure 4.

A copper bar is chosen as the work-piece and installed on the air spindle of the ultra-precision machining machine. The capacitive micro-displacement sensors with four measurement channels (Micro-sense DE 5300-013) are mounted on the handle of the apparatus to measure the vibration signals in three directions during cutting, the vibration signals are collected and then transmitted to the computer, which is used for data storage and signal processing. In addition, the power amplifier (PI, E-500), which has a criterion amplification factor 1060.1, is employed to amplify the driving signal. The Power PMAC controller generates the driving signals to drive the piezoelectric stack in the 
apparatus. The apparatus of 3D-EVC is driven by the piezoelectric hybrid, which, given the sinusoidal excitation signal, can be expressed as:

$$
\left\{\begin{array}{l}
x=A_{1} \sin \left(\omega_{1} t+\varphi_{1}\right) \\
y=A_{2} \sin \left(\omega_{2} t+\varphi_{2}\right) \\
z=A_{3} \sin \left(\omega_{3} t+\varphi_{3}\right)
\end{array}\right.
$$

where, $x, y$ and $z$ are the given sinusoidal excitation signal in $\mathrm{X}, \mathrm{Y}$ and $\mathrm{Z}$ directions of tool tip corresponding to the coordinate system in machine tools, respectively. $A_{1}, A_{2}$ and $A_{3}$ are the amplitudes of driving singles in three directions generated by three piezoelectric stacks, respectively. $t$ represents the time. In addition, $\varphi_{1}, \varphi_{2}$ and $\varphi_{3}$ are the angular frequency imposed on the piezoelectric stacks, and $\omega_{1}, \omega_{2}$ and $\omega_{3}$ are the phase of driving signal along $X, Y$ and $Z$ directions, respectively.

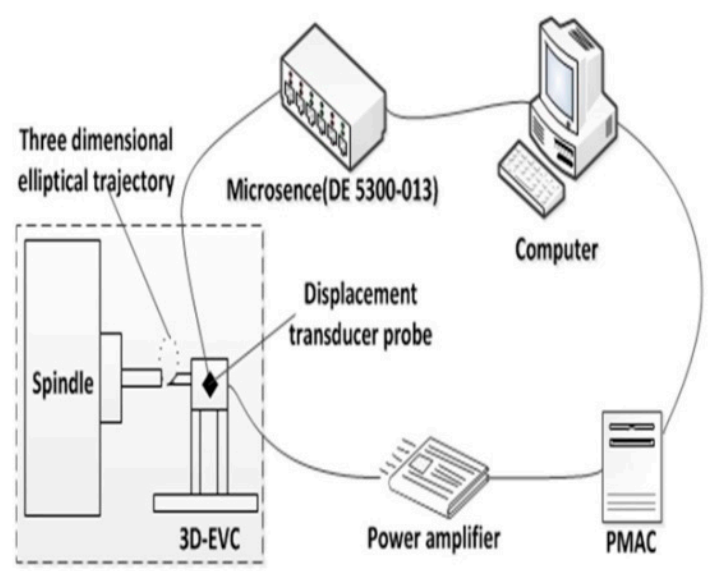

(a) Schematic diagram
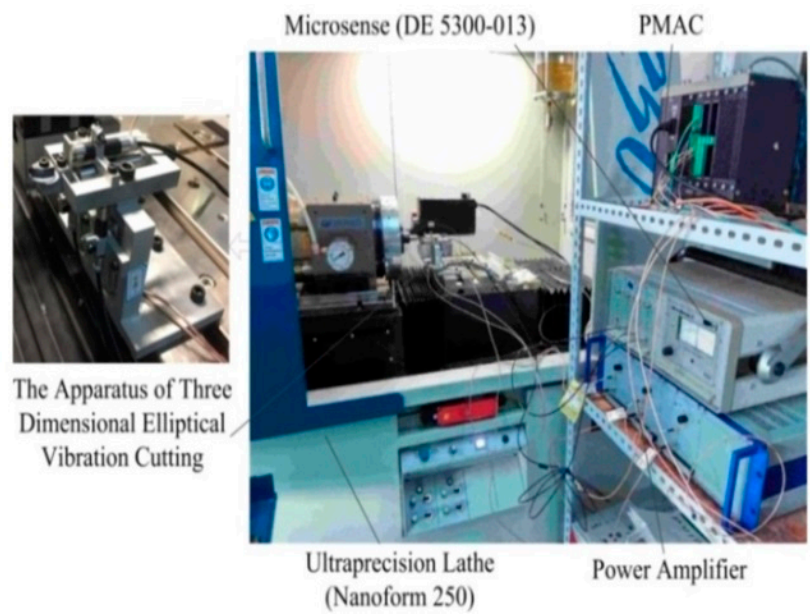

(b) Photo of the setup

Figure 4. Experimental setup.

In order to compare the different cutting states during the cutting process, the value of spindle speed and the feed rate is fixed $(90 \mathrm{rpm} / \mathrm{min}$ and $10 \mathrm{~mm} / \mathrm{min}$, respectively), the depth of cut is set to $5 \mu \mathrm{m}, 10 \mu \mathrm{m}$ and $20 \mu \mathrm{m}$, respectively. The cutting process was slotting with the oil mist cutting fluid. To avoid the phenomenon of overcut or the larger cutting marks between the two periods, the amplitude of piezoelectric driving signal is $6 \mu \mathrm{m}$. The detailed experimental conditions are listed in Table 1, and the main parameters of the material are listed in Table 2.

Table 1. Experimental conditions.

\begin{tabular}{ccc}
\hline & Parameter & Value \\
\hline \multirow{3}{*}{ Work-piece } & Material & Copper $(\mathrm{H62})$ \\
& Length $(\mathrm{mm})$ & 50 \\
& Diameter $(\mathrm{mm})$ & 12.7 \\
\hline \multirow{3}{*}{ Diamond tool } & Nose radius $(\mathrm{mm})$ & 0.2 \\
& Nominal rake angle $\left(^{\circ}\right)$ & 0 \\
& Nominal clearance angle $\left(^{\circ}\right)$ & 7 \\
\hline \multirow{3}{*}{ Cutting parameter } & Depth of cut $(\mu \mathrm{m})$ & $5,10,20$ \\
& Spindle speed $(\mathrm{rpm} / \mathrm{min})$ & 90 \\
& Feed rate $(\mathrm{mm} / \mathrm{min})$ & 10 \\
& vibration amplitude $(\mu \mathrm{m})$ & 6 \\
\hline
\end{tabular}


Table 2. Material properties.

\begin{tabular}{ccccc}
\hline Material & $\begin{array}{c}\text { Tensile Strength } \mathbf{R m} \\
\left(\mathbf{N} / \mathbf{m m}^{\mathbf{2}}\right)\end{array}$ & $\begin{array}{c}\text { Elongation to } \\
\text { Failure/(>\%) }\end{array}$ & Vickers Hardness & Density $\left(\mathbf{g} / \mathbf{c m}^{3}\right)$ \\
\hline Copper & $335-370$ & 24 & $85-128$ & 8.27 \\
\hline
\end{tabular}

\section{Results and Discussions}

\subsection{Initial Identification}

In the cutting process, there are two main characteristics to identify undesired vibrations: (1) the vibration amplitude increases gradually in the time domain as a result of the vibration energy increases; and (2) the frequency shift phenomenon will appear with the change of cutting the state in the frequency domain. This is one of the most significant signs to determine the occurrence of undesired vibration.

The time domain signal of three directions in the cutting process is collected by the displacement sensor, when the depth of cut is set to $5 \mu \mathrm{m}$, as shown in Figure 5. It can be seen from the time domain signals of three directions on the cutting tool that the vibration amplitudes along the $Z$ direction have greater values compared to the other two directions. This is because that the oscillation generated on the tool tip of 3D-EVC apparatus in Z direction contains more capacity. Consequently, vibration signals in $\mathrm{Z}$ direction are considered as the research object and further analysis in this paper.
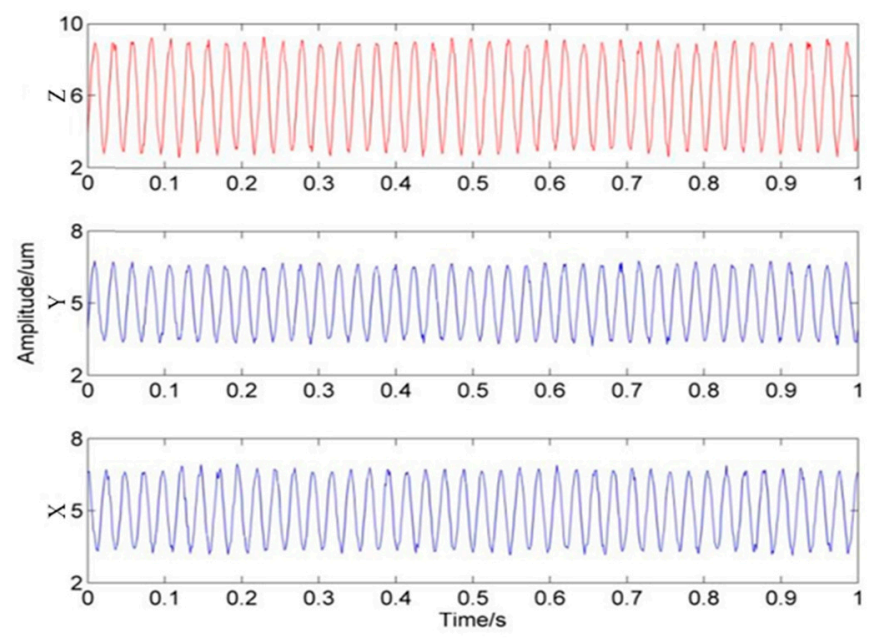

Figure 5. The time domain signal of three directions in 3D-EVC process (spindle speed was $90 \mathrm{rpm} / \mathrm{min}$, and the feed rate was $10 \mathrm{~mm} / \mathrm{min}$, slotting.

In order to obtain the different cutting states in 3D-EVC process, the original signals of vibration in $\mathrm{Z}$ direction are obtained under various cutting conditions (the depth of cut was $5 \mu \mathrm{m}, 10 \mu \mathrm{m}$ and $20 \mu \mathrm{m}$, respectively). The time domain signals in three kinds of cutting depth are shown in Figure $6 a-c$, the amplitude of vibration signals in frequency domain obtained by FFT are shown in Figure $7 \mathrm{a}-\mathrm{c}$, respectively.

Three typical states (stable cutting, transient state, and chatter state) in the cutting process of 3D-EVC can be obtain based on the variation of frequency with the varied of cutting depth. In the stable condition of the cutting process, the amplitude of vibration signal is relatively small and there is no larger peak or trough in Figure 6a; the frequency domain spectrum obtained by FFT is shown in Figure 7a. It is observed that the characteristics of the peaks appear at the frequency about $40 \mathrm{~Hz}$ corresponding to the natural frequency of 3D-EVC apparatus. In the condition of a transient state, some smaller fluctuations appeared in the time domain, and the amplitude of vibration grows, with a small change in Figure $6 b$, while the frequency domain spectrum has a significant change which appears as two peaks in $150 \mathrm{~Hz}$ and $200 \mathrm{~Hz}$, as shown in Figure 7b. But the amplitude is relatively small 
and there is no obvious change in the time domain. In the condition of a chatter state, the amplitude of vibration increases obviously, the change of amplitude has an effect on the three-dimensional elliptic trajectory of the tool-tip in Figure 6c. Moreover, the frequencies of the characteristic peaks at $150 \mathrm{~Hz}$ and $200 \mathrm{~Hz}$ are formed by the major components in the frequency spectrum, as shown in Figure 7c. In this stage, the cutting process is accompanied by harsh noise, and the work-piece appears obvious vibration mark. This is caused by the extrusion pressure between the tool-tip and the work-piece increasing with the rise of cutting depth.

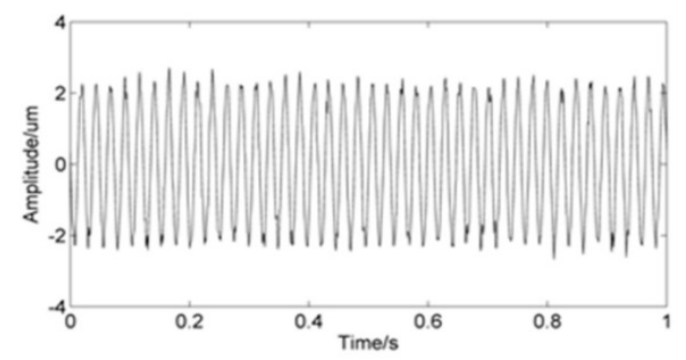

(a) Depth of cut is 5 um

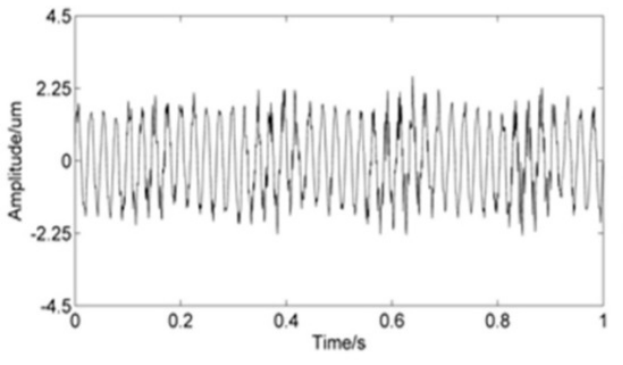

(b) Depth of cut is $10 \mathrm{um}$

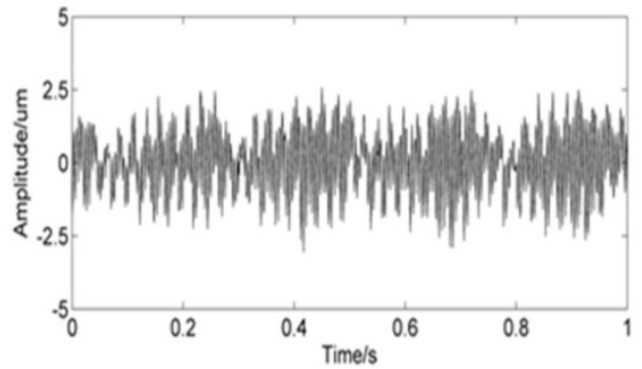

(c) Depth of cut is $20 \mathrm{um}$

Figure 6. Vibration displacement signal obtained in 3D-EVC process (spindle speed was $90 \mathrm{rpm} / \mathrm{min}$, the feed rate was $10 \mathrm{~mm} / \mathrm{min}$, misting).

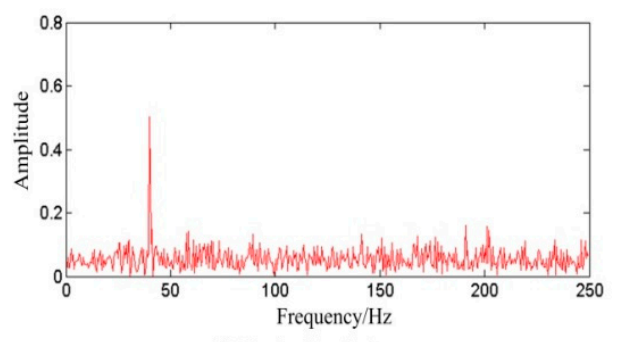

(a) Depth of cut is 5 um
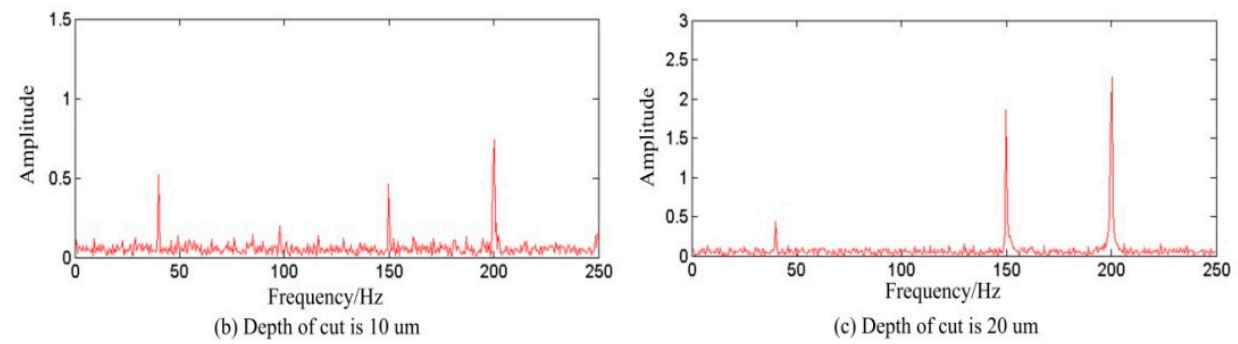

Figure 7. Frequency obtained by fast Fourier transform (FFT).

On the other hand, the cutting condition also affects the quality of the finished surface. Figure 8 shows quality of the finished surface under the three different conditions during the 3D-EVC process, respectively. The measurements of the surface roughness for the finished surface are implemented by using the ZYGO-Newview 8200, a white light interferometer, and the surface roughness $R_{a}$ of 
the finished surface is used to estimate the three cutting states under various cutting conditions. In the condition of stable cutting, as shown in Figure 8a, the finished surface has a clear and regular elliptical trajectory, owing to the small cutting parameters in 3D-EVC process, and the value of the surface roughness $R_{a}=0.046 \mu \mathrm{m}$. In the condition of a transient state, with the increase of cutting depth, the cycle of each elliptical motion starts to appear slight chatter marks, owing to the additional components corresponding to undesired vibration frequencies $(150 \mathrm{~Hz}$ and $200 \mathrm{~Hz})$ as shown in Figure $8 \mathrm{~b}$. Meanwhile, the value of surface roughness also increased with the augment of cutting depth $\mathrm{R}_{\mathrm{a}}=0.245 \mu \mathrm{m}$. In the condition of a chatter state, the elliptical motion trajectory becomes irregular in the finished surface, as shown in Figure $8 c$, the undesired vibration causes the discontinuous contact between the tool tip and the work-piece in the cycle of each elliptical motion, and the intermittent chopping-elliptical-vibration cutting is formed in the process of 3D-EVC, which corresponds to the large value of surface roughness $R_{a}=0.853 \mu \mathrm{m}$.

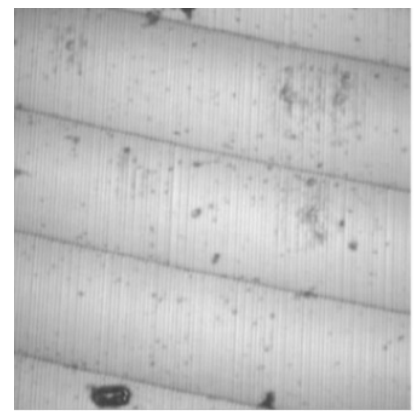

(a)

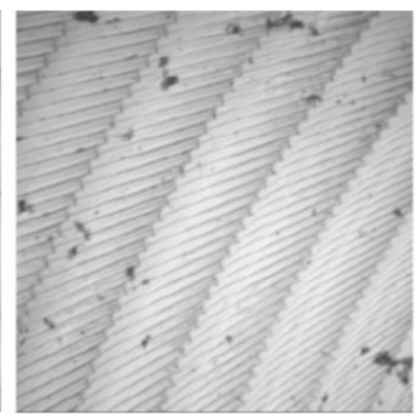

(b)

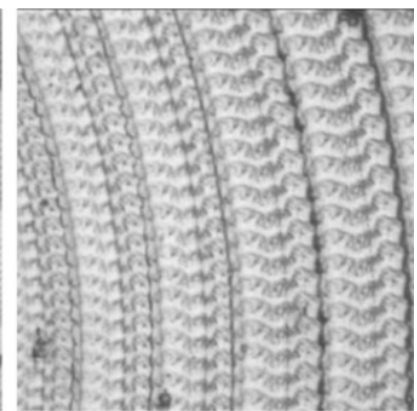

(c)

Figure 8. The quality of finished surface (a) Stable cutting (b) Transient state (c) Chatter state.

\subsection{Feature Extraction Identification}

In order to identify the chatter phenomenon accurately in the 3D-EVC process, feature extraction identification, or extracting the features of the chatter symptoms from the various cutting parameters, was proposed. Firstly, three groups of the original signals are decomposed with the EMD and a series of IMFs in stable cutting, transient and chatter state, are obtained. Then, the mean square frequency (MSF) and one step self-correlation coefficient $\rho_{1}$ of each IMF, which processes the primary energy in the 3D-EVC process, is calculated as the criteria for chatter identification. In this paper, the first six IMFs which contain mainly vibration information are selected, as shown in Figures 9-11, respectively.

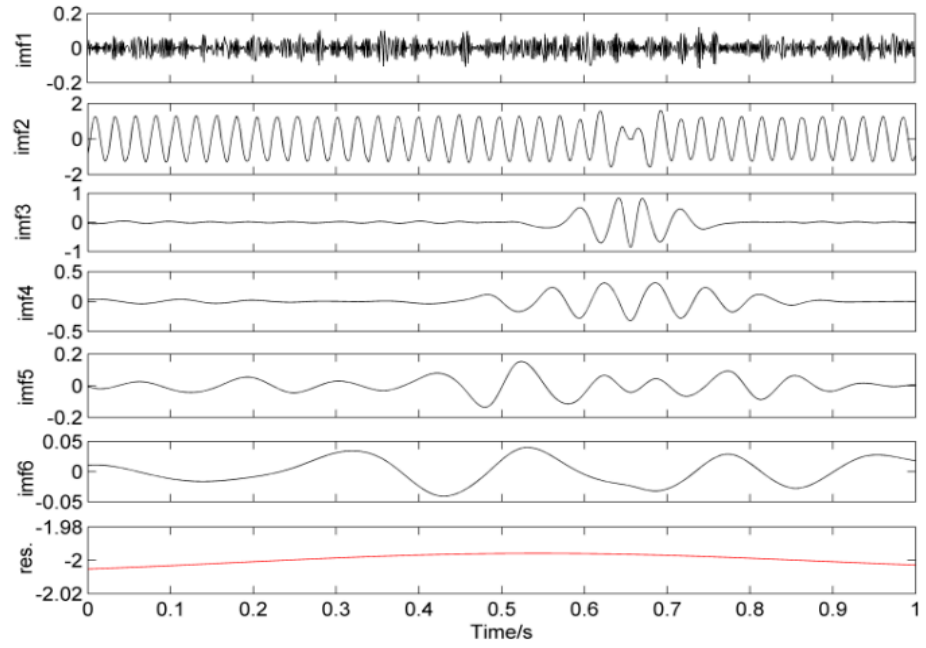

Figure 9. The result of EMD in stable cutting (spindle speed is $90 \mathrm{rpm} / \mathrm{min}$, the feed rate is $10 \mathrm{~mm} / \mathrm{min}$, and the depth of cut is $5 \mu \mathrm{m})$. 


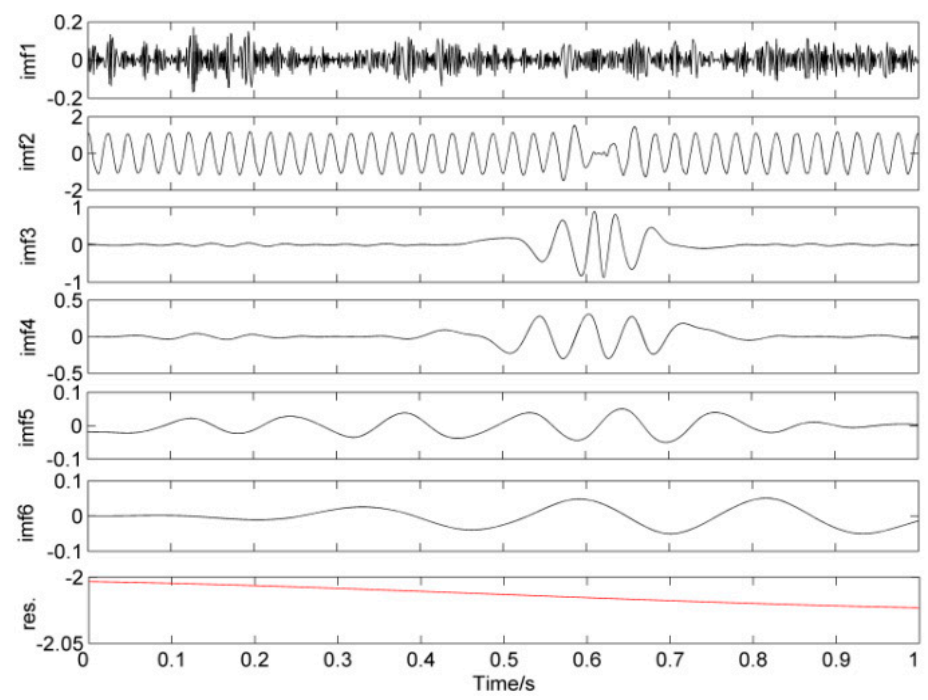

Figure 10. The result of EMD in transient state (spindle speed is $90 \mathrm{rpm} / \mathrm{min}$, the feed rate is $10 \mathrm{~mm} / \mathrm{min}$, and the depth of cut is $10 \mu \mathrm{m}$ ).

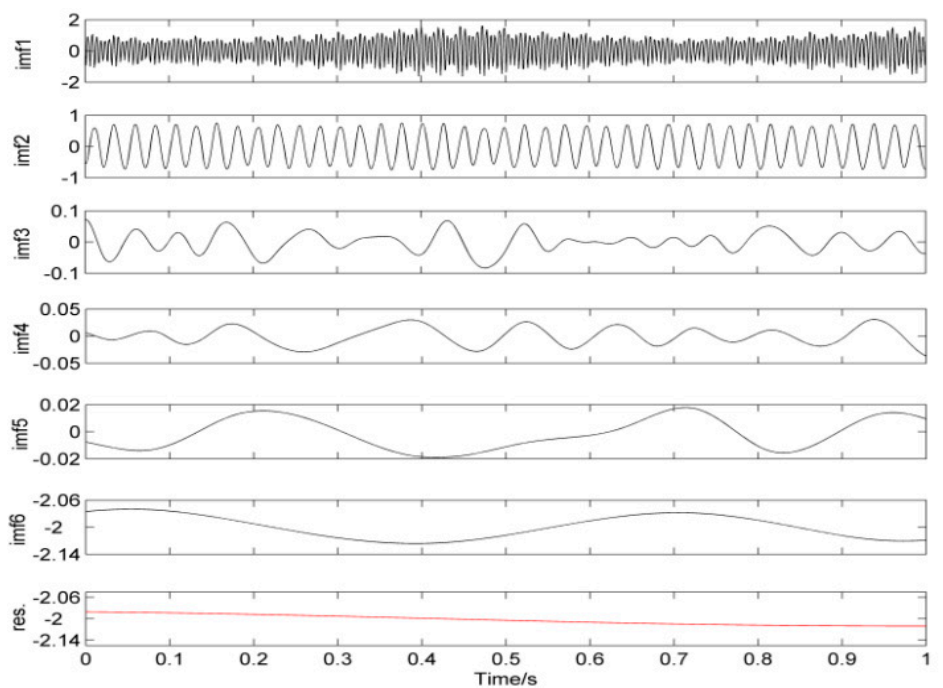

Figure 11. The result of EMD in chatter state (spindle speed is $90 \mathrm{rpm} / \mathrm{min}$, the feed rate is $10 \mathrm{~mm} / \mathrm{min}$, and the depth of cut is $20 \mu \mathrm{m}$ ).

The values of MSF and $\rho_{1}$ are calculated to judge the development trend of the vibration signals, and regarded as the index of the criterion for the undesired vibration in the 3D-EVC process, as listed in Table 3. As mentioned in the third section, MSF represents the weighted mean of the square value of the vibration frequency. When the undesired vibration happens suddenly, the frequency-shifting phenomenon will appear and the chatter frequency gradually increases. The value of MSF also increased with the cutting state growing fluctuant. In addition, $\rho_{1}$ also has consanguineous relationship with the variety of the energy in vibration signals. The value of $\rho_{1}$ will reduce suddenly when the energy concentrates around the chatter frequencies, as a result of the uneven amplitude of vibration signals. In a stable cutting process, the values of MSF and $\rho_{1}$ are 210 and 0.25 , this value represents a relatively stable state in the cutting process. For the transient state, the value of MSF increases to 270, moreover, the value of $\rho_{1}$ is reduced to 0.57 . In the chatter state, the value of MSF increases to 6800, this increased value is explained by the fact that the chatter frequency has turned into a significant component in the 3D-EVC process. However, such as in the above theoretical study, the value of $\rho_{1}$ is reduced to 0.29 , as the correlation between the vibration signals had reduced. Therefore, the numerical 
variation of MSF and $\rho_{1}$ can be applied to an effective criterion, which identifies the change of cutting state in the $3 \mathrm{D}-\mathrm{EVC}$ process.

Table 3. The value of mean square frequency (MSF) and $\rho_{1}$ under the three cutting conditions.

\begin{tabular}{ccc}
\hline Cutting Conditions & MSF & $\boldsymbol{\rho}_{1}$ \\
\hline Stable cutting & 210 & 0.95 \\
Transient state & 270 & 0.57 \\
Chatter state & 6800 & 0.29 \\
\hline
\end{tabular}

\section{Conclusions}

In this paper, a new chatter identification method based on signal processing was proposed to identify chatter phenomenon during the 3D-EVC process. The 3D vibration signals of the tool-tip are obtained by the displacement sensor, which is installed in the 3D-EVC apparatus, and the vibration signals were chosen as the object of decomposition, based on the visualized time and frequency domain data in the $\mathrm{Z}$ direction. The method of chatter identification developed is divided into two parts: initial identification and feature extraction identification. The amplitude spectrum obtained by FFT was used as a preliminary criterion for chatter identification. Then, the EMD method was employed to decompose the vibration signal including the rich undesired vibration information, the mean square frequency, and the one-step self-correlation coefficient, which were calculated as the identification criteria for identifying the chatter in the 3D-EVC process. The experimental results show:

(1) The vibration signals obtained by the displacement transducer and the amplitude spectrum obtained by FFT have reflected the trend of vibration state in the 3D-EVC process from the time and the frequency domain, respectively. The frequency shift phenomenon appeared with the vibration energy increasing gradually in the machining processes.

(2) Both the mean square frequency and the one step self-correlation coefficient have been changed suddenly in the cutting process. The value of the mean square frequency increases from 210 to 6800 , in contrast, the value of the one step self-correlation coefficient reduces from 0.95 to 0.29 . Therefore, these two parameters can be used as the index of the chatter phenomenon.

In future work, the effects of model parameters will be theoretically analyzed with stability lobe theory. Moreover, a chatter suppression method should be developed by combining sensorless chatter detection with an optimization algorithm.

Author Contributions: J.L., B.C. and M.L. conceived and designed the experiments; B.C., J.Z., and D.Z. performed the experiments; B.C., A.Y. and H.W. analyzed the data; J.L. and M.L. contributed reagents/materials/analysis tools; B.C. wrote the paper; M.L., B.C., J.Z. and D.Z. reviewed and revised the paper.

Funding: This research was funded by [Ministry of Science and Technology State Key Support Program] grant number [2016YFE0105100], [Micro-Nano and Ultra-Precision Key Laboratory of Jilin Province] grant number [20140622008JC], [Science and Technology Development Projects of Jilin Province] grant number [20180101034JC, 20180201052GX], [Education Department Scientific Research Planning Project of Jilin Provincial] grant number [JJKH20181038KJ] And the APC was funded by [20180101034JC].

Acknowledgments: Special thanks to the experimental installation provided by the Key Laboratory of Micro-Nano and Ultra-precision Manufacturing of Jilin Province.

Conflicts of Interest: The authors declare no conflict of interest.

\section{References}

1. Shamoto, E.; Moriwaki, T. Study on elliptical vibration cutting. CIRP Ann. Manuf. Technol. 1994, 43, 35-38. [CrossRef]

2. Shamoto, E.; Moriwaki, T. Ultaprecision diamond cutting of hardened steel by applying elliptical vibration cutting. CIRP Ann. Manuf. Technol. 1999, 48, 441-444. [CrossRef] 
3. Lu, M.; Zhou, J.; Lin, J.; Gu, Y.; Han, J.; Zhao, D. Study on Ti-6Aql-4V alloy machining applying the non-resonant three-dimensional elliptical vibration cutting. Micromachines 2017, 8, 306. [CrossRef] [PubMed]

4. Shamoto, E.; Suzuki, N.; Tsuchiya, E.; Hori, Y.; Inagaki, H.; Yoshino, K. Development of 3 DOF ultrasonic vibration tool for elliptical vibration cutting of sculptured surfaces. CIRP Ann. Manuf. Technol. 2005, 54, 321-324. [CrossRef]

5. Lu, M.; Zhao, D.; Lin, J.; Zhou, X.; Zhou, J.; Chen, B.; Wang, H. Design and analysis of a novel piezoelectrically actuated vibration assisted rotation cutting system. Smart Mater. Struct. 2018, 27, 095020. [CrossRef]

6. Quintana, G.; Ciurana, J. Chatter in machining processes: A review. Int. J. Mach. Tools Manuf. 2011, 51, 363-376. [CrossRef]

7. Siddhpura, M.; Paurobally, R. A review of chatter vibration research in turning. Int. J. Mach. Tools Manuf. 2012, 61, 27-47. [CrossRef]

8. Shamoto, E.; Suzuki, N.; Hino, R. Analysis of 3D elliptical vibration cutting with thin shear plane model. CIRP Ann. Manuf. Technol. 2008, 57, 57-60. [CrossRef]

9. Ma, C.; Ma, J.; Shamoto, E.; Moriwaki, T. Analysis of regenerative chatter suppression with adding the ultrasonic elliptical vibration on the cutting tool. Precis. Eng. 2011, 35, 329-338. [CrossRef]

10. Zhang, S.J.; To, S.; Zhang, G.Q.; Zhu, Z.W. A review of machine-tool vibration and its influence upon surface generation in ultra-precision machining. Int. J. Mach. Tools Manuf. 2015, 91, 34-42. [CrossRef]

11. Chen, C.C.; Liu, N.M.; Chiang, K.T.; Chen, H.L. Experimental investigation of tool vibration and surface roughness in the precision end-milling process using the singular spectrum analysis. Int. J. Adv. Manuf. Technol. 2012, 63, 797-815. [CrossRef]

12. Chen, C.C.; Chiang, K.T.; Chou, C.C.; Liao, Y.C. The use of D-optimal design for modeling and analyzing the vibration and surface roughness in the precision turning with a diamond cutting tool. Int. J. Adv. Manuf. Technol. 2011, 54, 465-478. [CrossRef]

13. Liu, C.; Zhu, L.; Ni, C. The chatter identification in end milling based on combining EMD and W PD. Int. J. Adv. Manuf. Technol. 2017, 91, 3339-3348. [CrossRef]

14. Lamraoui, M.; Thomas, M.; El Badaoui, M.; Girardin, F. Indicators for monitoring chatter in milling based on instantaneous angular speeds. Mech. Syst. Signal Process. 2014, 44, 72-85. [CrossRef]

15. Tangjitsitcharoen, S.; Saksri, T.; Ratanakuakangwan, S. Advance in chatter detection in ball end milling process by utilizing wavelet transform. J. Intell. Manuf. 2015, 26, 485-499. [CrossRef]

16. Rafal, R.; Pawel, L.; Krzysztof, K.; Bogdan, K.; Jerzy, W. Chatter identification methods on the basis of time series measured during titanium superalloy milling. Int. J. Mech. Sci. 2015, 99, 196-207. [CrossRef]

17. Thaler, T.; Potočnik, P.; Bric, I.; Govekar, E. Chatter detection in band sawing based on discriminant analysis of sound features. Appl. Acoust. 2014, 77, 114-121. [CrossRef]

18. Hynynen, K.M.; Ratava, J.; Lindh, T.; Rikkonen, M.; Ryynänen, V.; Lohtander, M.; Varis, J. Chatter detection in turning processes using coherence of acceleration and audio signals. J. Manuf. Sci. Eng. 2014, 136, 044503. [CrossRef]

19. Lamraoui, M.; Thomas, M.; El Badaoui, M. Cyclostationarity approach for monitoring chatter and tool wear in high speed milling. Mech. Syst. Signal Process. 2014, 44, 177-198. [CrossRef]

20. Cao, H.; Lei, Y.; He, Z. Chatter identification in end milling process using wavelet packets and Hilbert-Huang transform. Int. J. Mach. Tools Manuf. 2013, 69, 11-19. [CrossRef]

21. Cao, H.; Zhou, K.; Chen, X. Chatter identification in end milling process based on EEMD and nonlinear dimensionless indicators. Int. J. Mach. Tools Manuf. 2015, 92, 52-59. [CrossRef]

22. Jung, H.; Hayasaka, T.; Shamoto, E. Mechanism and suppression of frictional chatter in high-efficiency elliptical vibration cutting. CIRP Ann. 2016, 65, 369-372. [CrossRef]

23. Vela-Martínez, L.; Jáuregui-Correa, J.C.; Rubio-Cerda, E.; Herrera-Ruiz, G.; Lozano-Guzmán, A. Analysis of compliance between the cutting tool and the workpiece on the stability of a turning process. Int. J. Mach. Tools Manuf. 2008, 48, 1054-1062. [CrossRef]

24. Huang, N.E.; Shen, Z.; Long, S.R.; Wu, M.C.; Shih, H.H.; Zheng, Q.; Yen, N.C.; Tung, C.C.; Liu, H.H. The empirical mode decomposition and the Hilbert spectrum for nonlinear and non-stationary time series analysis. R. Soc. 1998, 454, 903-995. [CrossRef] 
25. Saidi, L.; Ali, J.B.; Fnaiech, F. Bi-spectrum based-EMD applied to the non-stationary vibration signals for bearing faults diagnosis. ISA Trans. 2014, 53, 1650-1660. [CrossRef] [PubMed]

26. Li, X.; Mei, D.Q.; Chen, Z.C. Feature extraction of chatter for precision holes boring processing based on EMD and HHT. Opt. Precis. Eng. 2011, 19, 1291-1297. 